\title{
Efficacy of Enhanced External Counterpulsation (EECP) in Nepalese chronic stable Angina patient: a single centre prospective study at Shahid Gangalal National Heart Center (SGNHC).
}

\author{
Adhikari $\mathrm{CM}^{1}$,Prajapati $\mathrm{D}^{1}$, Thapa $\mathrm{S}^{2}, \mathrm{KC} \mathrm{MB}^{1}$ \\ 1 Department of Cardiology, SGNHC, Bansbari, Kathmandu, Nepal. \\ 2 Department of Cardiac Rehabilation and Health Promotion, SGNHC, Bansbari, Kathmandu, Nepal.
}

\begin{abstract}
Background

Enhanced External Counterpulsation (EECP) is a novel; FDA approved; non-invasive; outpatient treatment offered to patients with refractory angina pectoris. It uses sequentially inflated pneumatic cuffs on the lower extremities to enhance coronary diastolic flow. We studied its effect in twenty one patients with refractory angina pectoris.
\end{abstract}

Methods

All patients $(n=21)$ who were referred for Enhanced External Counterpulsation to Shahid Gangalal National Heart Center Enhanced External Counterpulsation Llb who completed a treatment course (one hour per day for 35 days) of EECP and underwent 6-minute walk test before and after treatment were included. Demographic data, coronary artery disease risk factors and baseline angiographic data were collected. Distance covered in six minute walk test before and after the treatment was compared.

Results

All the patients who had undergone Enhanced External Counterpulsation had a positive clinical response. Distance covered in six minute walk test improved in all patients after the treatment. Decrease in anginal severity, frequency and the use of sublingual nitrates, with improvement in quality of life was observed after the treatment. During the treatment some patients complained of leg pain, one patient developed blister and one ecchymosis but the treatment was not discontinued.

Conclusion

The results from this study suggest that Enhanced External Counterpulsation is an effective, safe and well tolerated treatment option for the patients with refractory angina pectoris.

Keywords Enhanced External Counterpulsation, Refractory angina pectoris 


\section{Introduction}

Refractory angina pectoris (RAP) is a clinical diagnosis which is characterized by chronic angina in patients who are refractory to conventional treatment. ${ }^{1}$ Treatment of chronic stable angina consists of pharmacological interventions and invasive actions such as percutaneous coronary interventions $(\mathrm{PCl})$ and coronary bypass grafting (CABG). Despite of these generally successful means of treatment the number of patients with severe symptomatic ischemic chest pain has increased. ${ }^{2}$ Some patients cannot undergo $\mathrm{PCl}$ and CABG due to diffuse coronary artery stenosis. It has been reported that up to $15 \%$ of patients with angina pectoris meet the criteria for refractory angina. ${ }^{3}$ To cope of with this significant clinical problem, a lot of alternative therapies evolved. Among them, EECP therapy is the most promising treatments for relieving angina and has been shown to improve exercise tolerance in patients with symptoms of stable angina pectoris. ${ }^{4} \mathrm{EECP}$ is a non-invasive, pneumatic technique that can be offered to patients with angina refractory to anti-anginal medications, who are not suitable candidates for conventional revascularization procedures. $^{5}$

In EECP, three sets of pneumatic cuffs are wrapped around the lower extremities. The cuffs are inflated sequentially at the onset of diastole, producing aortic counter pulsation, diastolic augmentation, and increased venous return. At the onset of systole, the external pressure in the cuffs is released, producing a decrease in systolic pressure. This rapid inflation and deflation raises diastolic aortic pressure, increases coronary perfusion pressure, provides afterload reduction, and enhances venous return with a subsequent increase in cardiac output. ${ }^{4}$ The hemodynamic effects are similar to intra-aortic balloon pumping (IABP). ${ }^{6,7} \mathrm{~A}$ treatment procedure involves one to two hours/day for a total of 35 hours of therapy. Several studies around the world have shown patient improvement with lowering in Canadian Cardiovascular Society Classification (CCS) angina class. ${ }^{8,9}$ In addition to relieving myocardial ischemia, EECP is associated with improved quality of life. ${ }^{9,10}$

The aim of the present study was to evaluate the efficacy of EECP treatment in Nepalese chronic stable angina patients. The study was designed to examine the immediate effects on chronic stable angina patients who are considered unsuitable for revascularization and are refractory to optimal medical management.

\section{Methods}

Enhanced external counter pulsation equipment is supplied by the manufacturer; Vamed Company (Guangzhou, China).The equipment consists of an air compressor, a console, a treatment table and two sets of three cuffs. Before a treatment session, cuffs are wrapped around the patient's legs, one set on each leg. Using compressed air, pressure $(260-350 \mathrm{mmHg}$ of external pressure) was applied via the cuffs to the patient's lower extremities in a sequence synchronized with the cardiac cycle. In early diastole, pressure was applied sequentially from the lower legs to the lower and upper thighs to propel blood back to the heart. This resulted in an increase of arterial blood pressure and retrograde aortic blood flow during diastole (diastolic augmentation).At end-diastole, air was released instantaneously from all the cuffs to remove the externally applied pressure, allowing the compressed vessels to reconfirm, thereby reducing vascular impedance. Blood pressure changes were monitored by finger plethysmography. Daily one hour treatment sessions were administered for a total treatment course of 35 hours.

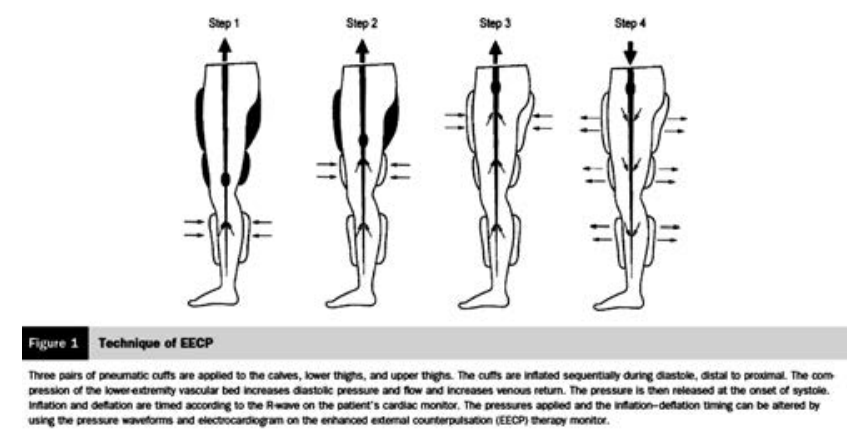

\section{Study population and protocol}

All twenty one $(n=21)$ consecutive patients who underwent 35 hours of EECP and a six minute walk test before and after the treatment in Shahid Gangalal National heart Centre from 2010 were enrolled in the study. The protocol was approved by the Ethics Committee of the Shahid Gangalal National heart Centre. Informed consent was taken from patient and patient party. Patients' data, which had been recorded prior to treatment, included age, gender, past history of diabetes mellitus (DM), hypertension, dyslipidaemia, cigarette smoking and previous angiographic data (angiographic score as Single (SVD)-, two(DVD)-or three(TVD)-vessel disease).All the contraindication to EECP therapy were ruled out in each patient. All patients underwent six minute walk test before and after the completion of the 35 hours of EECP. Patients were also followed with the questionnaires about their anginal symptoms after the completion of treatment.

\section{Six minute walk test}

A six minute walk test was performed under the same circumstances for all the patients at baseline and at the end of the treatment by an independent observer. The distance covered in six minute was recorded and compared. 


\section{Statistical analysis}

The two-tailed paired $t$-test was used to evaluate the significance of improvement in the distance covered in six minute walk test before and after the treatment.

\section{Results}

Twenty one patients completed 35 hours of treatment within seven weeks. Table-1 shows the demographic and clinical characteristics. The mean age was $67.1 \pm 10.4$ years. Of them 11 were male and 10 female, 15 with CAD Triple vessel disease, six with CAD double vessel disease, four patient undergone $C A B G$ and one underwent $\mathrm{PCl}$ stenting to two vessels. Nineteen were hypertensive, nine were diabetics, eight were smoker before. Low LVEF defined as LVEF less than $40 \%$ was present in one patient. EECP was chosen as treatment due to angina refractory to medical management and patient not suitable for CABG and $\mathrm{PCl}$.

Table 1: Baseline Demographic and Clinical characteristics: $\mathrm{N}=$ $21(\%)$

\begin{tabular}{|c|c|}
\hline Mean Age & $67.1 \pm 10.4$ years \\
\hline Male /Female & $(52 / 48)$ \\
\hline \multicolumn{2}{|l|}{ Medical history } \\
\hline Hypertension & (90) \\
\hline Diabetes & (42) \\
\hline Ex-smoker & (38) \\
\hline Dyslipidemia & All patient taking statin \\
\hline CAD TVD & (72) \\
\hline CAD DVD & (28) \\
\hline Post CABG & (19) \\
\hline Post PCl & (4.7) \\
\hline Post CABG+Post PCI & (4.7) \\
\hline Low LVEF (LVEF<40\%) & (4.7) \\
\hline
\end{tabular}

During six minute walk test there was improvement in the distance covered (table 2).

Table 2: Comparison of 6 min walk test distance before and after EECP.

\begin{tabular}{|l|c|c|l|} 
& $\begin{array}{r}\text { Pre EECP } \\
\text { Feet } \pm \text { SD }\end{array}$ & $\begin{array}{c}\text { Post EECP } \\
\text { Feet } \pm \text { SD }\end{array}$ & P value \\
\hline 6 min walk test distance & $835.4 \pm 389.9$ & $1103.5 \pm 371.9$ & $<0.001$ \\
\hline
\end{tabular}

Table 3: Comparison of 6 min walk test distance before and after EECP in each patient

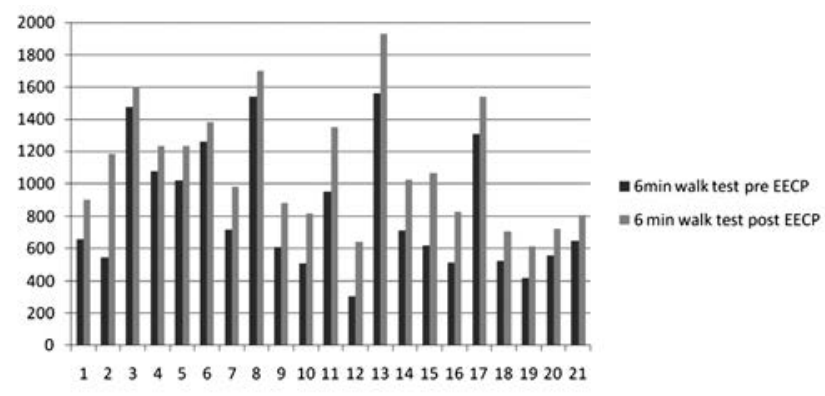

Based on the questionnaires about their anginal symptoms all the patient had decreased in severity of angina, decreased in the frequency of angina, decreased in sublingual nitrates use.

\section{Discussion}

The present study is the continuation of our previous study published as an abstract on Nepalese Heart Journal 2011 on efficacy of EECP in Nepal for chronic stable angina pectoris patient refractory to conventional therapy. The majority of the patients showed a profile of extensive coronary artery disease and poor quality of life. These patients' coronary arteries were not suitable for revascularization and were on optimal pharmacological treatment. The medical regimen was not changed during the EECP treatment.

The results from the present study confirm that EECP treatment significantly increases the distance covered in six minute walk test in chronic stable angina pectoris patient, which is in accordance with international studies. ${ }^{11,12}$ It was noted that there was a significant decrease in the frequency of angina episodes and nitroglycerin usage based on patient history.

The MUST-EECP (Multicentre Study of Enhanced External Counter Pulsation), the first and only multicentre, prospective, randomized, and blinded, placebo controlled trial on the subject, assessed the efficacy of EECP. In this trial, patients undergoing active counter-pulsation had a significant decrease in angina episodes, but there was no significant improvement in the duration of the exercise test. ${ }^{4}$ But relief in myocardial ischemia as well as improved quality of life has been shown in a number of studies from different countries. ${ }^{13}$

In one study, Lawson et al. studied 50 patients with chronic stable angina and compared the extent of coronary disease with results of radionuclide stress testing after EECP. This study showed significant improvement in the perfusion defects after EECP and the less the coronary disease involvement, the greater the therapeutic benefit from EECP. ${ }^{14}$

In a report of data from an EECP consortium which included 2,284 patients, an improvement was reported in up to $74 \%$ of patients with angina undergoing EECP, by one or more CCS functional classes. The younger patients had a greater likelihood of improvement. ${ }^{11}$

EECP increases diastolic aortic pressure, reduces systolic pressure and enhances venous return, thus resulting in increased cardiac output.$^{15}$ However, the mechanisms by which these hemodynamic effects lead to a reduction of angina are poorly understood, although the effect is similar to IABP.7 There is accumulating evidence suggesting that 
EECP treatment improves endothelial function, which may contribute to the clinical benefit . ${ }^{8} \mathrm{EECP}$ treatment is associated with an immediate increase in blood flow in multiple vascular beds including the coronary arterial circulation. ${ }^{7}$ This increase in blood flow may result in increased endothelial shear stress, ${ }^{16}$ which enhances endothelial function by stimulating the release of the vasodilatory mediator nitric oxide and reduces the release of the vasocontractile endothelin-1. ${ }^{15,17-19}$ However, most of the experience is from small animal or human uncontrolled studies, and the mechanism of the sustained antianginal benefit with EECP remains unclear.

Long term effects of EECP treatment are thought to be mediated through shear stress on the vascular endothelium, which in turn triggers angiogenesis and improves vascular endothelial function ${ }^{20}$ due to modulated release of vasoactive substances such as endothelin, ${ }^{21}$ nitric oxide ${ }^{21}$ and vascular endothelial growth factor. ${ }^{22}$

Furthermore, besides the release of metabolites from ischemic regions, an increase in endothelial shear stress is considered a major stimulus for collateral blood vessel development and recruitment. ${ }^{22}$ Increase in collateral perfusion is by opening preformed collateral channels, either directly via increasing diastolic blood pressure and flow or indirectly via release of vasodilator mediators. ${ }^{8}$

EECP therapy has been associated with the release of angiogenic factors, such as vascular endothelial growth factor, ${ }^{22}$ basic fibroblast growth factor and hepatocyte growth factor. ${ }^{21}$ In recent studies by Tao et $\mathrm{al}^{20,23}$
EECP therapy demonstrated stabilization of coronary endothelium, an effect very similar to that of athletic training. Lawson et al reported that EECP seemed to exert a training effect, decreasing peripheral vascular resistance and the heart rate response to exercise. ${ }^{24}$

\section{Figure2: Mechanism of Action of EECP clinical benefit.}

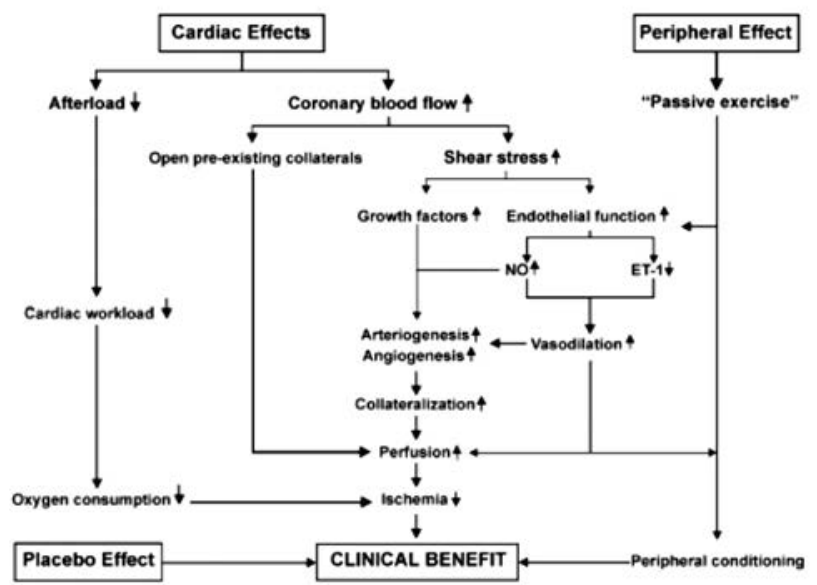

\section{Conclusion:}

The present study confirms EECP as a safe and practical treatment for angina in general clinical practice. Its more widespread availability as a treatment alternative may prove beneficial to patient care. One limitation of our study was that the placebo effect of the device could not be ruled out

\section{References:}

1. Mannheimer C, Camici P, Chester MR, et al. The problem of chronic refractory angina; report from the ESC Joint Study Group on the Treatment of Refractory Angina. Eur Heart J 2002;23:355-70.

2. Yang EH, Barsness GW, Gersh BJ, et al. Current and future treatment strategies for refractory angina. Mayo Clin Proc 2004;79:1284-92.

3. Mannheimer C. Therapeutic challenges of refractory angina pectoris. In: XXth Congress of the European Society of.Cardiology, Vienna, Austria. 1998.

4. Arora RR, Chou TM, Jain D, et al, The multicenter study of enhanced external counterpulsation (MUST-EECP): effect of EECP on exercise induced myocardial ischemia and anginal episodes. J Am Coll Cardiol 1999;33:1833-40.

5. Shea ML, Conti CR, Arora RR. An update on enhanced external counterpulsation.Clin Cardiol 2005;28(3):115-8.
6. Taguchi I, Ogawa K, Kanaya T, et al. Effects of enhanced external counterpulsation on hemodynamics and its mechanism. Circ J 2004;68:1030-4.

7. Michaels AD, Accad M, Ports TA, et al. Left ventricular systolic unloading and augmentation of intracoronary pressure and Doppler flow during enhanced external counterpulsation. Circulation 2002;106:1237-42.

8. Bonetti PO, Holmes DRJ, Lerman A, et al. Enhanced external counterpulsation for ischemic heart disease: what's behind the curtain? J Am Coll Cardiol 2003;41:1918-1925.

9. Michaels AD, Linnemeier G, Soran O, et al. Two year outcomes after enhanced external counterpulsation for stable angina pectoris (from the International EECP Patient Registry [IEPR]). Am J Cardiol 2004;93:461-4.

10. Springer S, Fife A, Lawson W, et al. Psychosocial effects of enhanced external counterpulsation in the angina patient: a second study. Psychosomatic 2001;42:124-32. 
11. Lawson WE, Hui JC, Lang G. Treatment benefit in the enhanced external counterpulsation consortium. Cardiology 2000;94:31-5.

12. Stys T, Lawson WE, Hui JC, et al: Acute hemodynamic effects and angina improvement with enhanced external counterpulsation. Angiology 2001;52:653-8.

13. Arora RR, Chou TM, Jain D,et al: Effects of enhanced external conterpulsation on Health-Related Quality of Life continue 12 months after treatment: a substudy of the Multicenter Study of Enhanced External Counterpulsation. J Investig Med 2002;50:25-35.

14. Lawson WE, Hui JC, Cohn PF. Long-term prognosis of patients with angina treated with enhanced external counterpulsation: five-year follow-up study. Clin Cardiol 2000;23:254-8.

15. Barsness GW. Enhanced external counterpulsation in unrevascularizable patients. Curr Interv Cardiol Rep 2001;3:37-43.

16. Kern MJ, Aguirre FV, Tatineni S, et al: Enhanced coronary blood flow velocity during intraaortic balloon counterpulsation in critically ill patients. J Am Coll Cardiol 1993;21:359-68.

17. Kuchan MJ, Frangos JA. Shear stress regulates endothelin-1 release via protein kinase $C$ and $c$ GMP in cultured endothelial cells. Am J Physiol 1993;264:H150-6.

18. Davies PF. Flow-mediated endothelial echanotransduction. Physiol Rev 1995;75:519-60.
19. Masuda D, Nohara R, Hirai T, et al. Enhanced external counterpulsation improved myocardial perfusion and coronary flow reserve in patients with chronic stable angina; evaluation by (13) $\mathrm{N}$-ammonia positron emission tomography. Eur Heart J 2001;22:1451-8.

20. Bonetti PO, Barsness GW, Keelan PC, et al: Enhanced external counterpulsation improves endothelial function in patients with symptomatic coronary artery disease. J Am Coll Cardiol 2003;41:1761-8.

21. Akhtar M, Wu GF, Du ZM, et al: Effect of external counterpulsation on plasma nitric oxide and endothelin-1 levels. Am J Cardiol 2006;98:28-30.

22. Kersten JR, Pagel PS, Chilian WM, et al. Multifactorial basis for coronary collateralization: a complex adaptive response to ischemia. Cardiovasc Res 1999;43:44-57.

23. Tao J, Tu C, Yang Z, Zhang $Y$, et al. Enhanced external counterpulsation improves endothelium-dependent vasorelaxation in the carotid arteries of hypercholesterolemic pigs. Int J Cardiol 2006;112:269 -74.

24. Lawson WE, Hui JC, Zheng ZS, et al. Improved exercise tolerance following enhanced external counterpulsation: cardiac or peripheral effect? Cardiology 1996;87:271-5. 